\title{
Glottopol
}

Revue de sociolinguistique en ligne

$35 \mid 2021$

La langue à l'école, de l'institution à la classe : quelles conceptions, quelles normes, pour quels usages?

\section{La langue basque dans les instructions officielles (1976-2019)}

\section{Argia Olçomendy}

\section{(2) OpenEdition \\ Journals}

Édition électronique

URL : https://journals.openedition.org/glottopol/306

DOI : $10.4000 /$ glottopol.306

ISSN : 1769-7425

Éditeur

Presses universitaires de Rouen et du Havre

Référence électronique

Argia Olçomendy, "La langue basque dans les instructions officielles (1976-2019) », Glottopol [En

ligne], 35 | 2021, mis en ligne le 01 janvier 2021, consulté le 02 octobre 2021. URL : http://

journals.openedition.org/glottopol/306 ; DOI : https://doi.org/10.4000/glottopol.306

Glottopol 


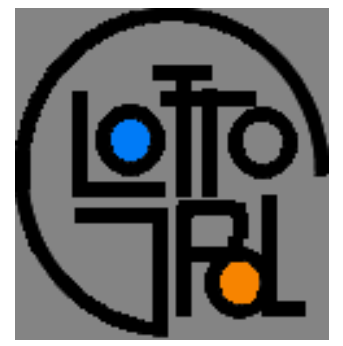

\section{GLOTTOPOL}

Revue de sociolinguistique en ligne $\mathrm{n}^{\circ} 35$ - janvier 2021

\section{La langue à l'école, de l'institution à la classe : quelles conceptions, quelles normes, pour quels usages?}

\section{SOMMAIRE}

Catherine Delarue-Breton et Élisabeth Bautier : Introduction

Catherine Delarue-Breton : Des conceptions scolaires de la langue en tension : l'exemple du programme de 2015 pour l'école primaire et le collège

Véronique Miguel-Addisu : "Sport de riche je l'ai changé en sport élitiste » : plurilinguisme et variation dans la langue de l'école du point de vue des élèves

Claire Colombel-Teuira, Véronique Fillol : Variation et approche polynomique : pour une conception plurielle de la langue à l'école calédonienne

Daphné Bloch : Pratiques langagières, situations pédagogiques et construction d'inégalités d'apprentissage à Madagascar

Samatar Abdallah Doualeh : Le français langue de l'école djiboutienne ou l'hégémonie de la norme exogène

Élise Vinel, Élisabeth Bautier : Des discours des élèves sur l'orthographe aux pratiques des enseignants, analyse d'entretiens métagraphiques

Thierry Pagnier, Belinda Lavieu-Gwozdz: Regards sur le discours scolaire: saisir des conceptions de la langue et de son enseignement

Jacques Crinon, Georges Ferone, Hélène Font : Les enseignants et l'orthographe, une enquête au cycle 3

Christel Troncy : Les enseignants, la norme scolaire et la pluralité langagière dans deux écoles immersives à programme français en Californie. Dynamiques des attitudes et des pratiques lors d'une recherche collaborative

Erwan Le Pipec: L'école, planche de salut du breton?

Argia Olçomendy : La langue basque dans les instructions officielles (1976-2019)

\section{Compte rendu de lecture}

Par Katrin Pfadenhauer: Ursula Reutner (Ed.), 2017, Manuel des francophonies, Berlin/Boston, de Gruyter, 745 p.

Par Robert Fournier: Shana Poplack, 2018, Borrowing: Loanwords in the speech community and in the grammar. New York: Oxford University Press ; xxi, $246 \mathrm{p}$.

Par Marie-Jeanne Verny: Catherine Adam, 2020, Bilinguisme scolaire. Familles, écoles, identités en Bretagne, éd. Peter Lang, coll. «Langue, multilinguisme et changement social », Berlin. 


\title{
LA LANGUE BASQUE DANS LES INSTRUCTIONS OFFICIELLES
} (1976-2019)

\author{
Argia Olçomendy \\ Université Bordeaux-Montaigne, IKER UMR 5478
}

\section{Introduction}

En France, chaque discipline scolaire définit les contenus de ses programmes scolaires fixés par des inspecteurs généraux et des spécialistes disciplinaires. La réflexion sur les programmes concerne les corps d'inspection de l'Éducation nationale comme les experts universitaires auxquels peuvent être associés d'autres représentants sociaux. Dans le cas de l'enseignement du basque, il existe des représentations collectives à propos de la complexité de la langue, bien souvent considérée comme étant source de difficultés d'apprentissage pour les élèves. La distance typologique entre le français/l'espagnol et le basque peut également freiner le processus d'apprentissage de la langue minorée d'un élève même après plusieurs années de scolarisation en basque. La différenciation entre savoirs linguistiques et pratiques langagières peut être source d'interrogations. D'autre part, le statut de la langue basque, langue transfrontalière est différent en fonction du territoire, mais à l'instar du catalan, la langue basque bénéficie d'une politique linguistique éducative menée dans la communauté autonome d'Euskadi qui pourrait nourrir la réflexion en Pays basque de France ${ }^{1}$.

Quelles sont les représentations et conceptions de langue basque véhiculées par les instructions officielles sur les langues régionales entre 1976 et 2019 ? D'après les programmes officiels, quelles sont les images sociales de la langue et quelle est la langue basque que les élèves suivant un cursus bilingue doivent maitriser? Tout d'abord, nous définirons un cadre théorique à notre étude pour ainsi approfondir les notions de représentation et de conception. Ensuite, nous indiquerons la méthodologie de recherche avec laquelle nous avons procédé pour enfin mettre en évidence les dimensions communicatives, métalinguistiques, littéraires et culturelles du discours institutionnel et proposer de nouvelles conceptions de la langue basque à l'école.

\footnotetext{
${ }^{1}$ La constitution espagnole du 27 décembre 1978 dit dans son article 3 : « 1. Le castillan est la langue espagnole officielle de l'État. Tous les Espagnols ont le devoir de le connaitre et le droit de l'utiliser. 2. Les autres langues espagnoles sont aussi officielles dans leurs communautés autonomes respectives conformément à leurs statuts ». En Euskadi, le Statut d'Autonomie du Pays Basque du 18 décembre 1979 dit dans son article 6-1 : «L'euskara, langue propre du peuple basque, a, comme le castillan, le caractère de langue officielle en Euskadi, et tous les habitants ont le droit de connaitre et d'employer les deux langues ». En Navarre, le Statut d'autonomie du 10 août 1982 stipule dans son article 9-1 que « le castillan est la langue officielle de la Navarre » et dans le 9-2 « que le basque a aussi le statut de langue officielle dans les régions basques de la Navarre » (Coyos, 2012).
} 


\section{Cadrage théorique}

La notion de représentation issue de la psychologie sociale et de la sociologie fait référence à des croyances construites par les membres de la communauté linguistique (Boyer, 2003 ; Lüdi et Py, 2003). Les lois et politiques linguistiques sont bien souvent conditionnées par les représentations sociales des langues (Agresti et Turi, 2016). Les représentations influencent la façon dont chaque groupe perçoit la réalité, tout élément de la réalité faisant l'objet d'une ou de plusieurs représentations partagées par la communauté ou le groupe d'appartenance de cet individu. Il en est alors de même pour les langues et les pratiques langagières qui n'échappent pas à la construction simplificatrice et schématique des représentations. C'est pourquoi cette notion est aujourd'hui largement prise en compte par les sociolinguistes. Pour comprendre l'insécurité linguistique, il est important de prendre en considération le rôle et l'intérêt des représentations et des stéréotypes (Labov, 1976). Le concept anglais «language attitude » comprend les deux concepts français, attitudes et représentations : « les attitudes envers la langue sont d'une extrême uniformité au sein d'une communauté linguistique » (Labov : 1976, 338). Au Pays basque, les jeunes enseignants sont confrontés à l'insécurité linguistique caractérisée par un sentiment d'échec dans la tentative d'appropriation et d'utilisation de la norme dominante.

Les représentations sociolinguistiques sont «une catégorie de représentations sociales / collectives, partagées par les membres de la communauté linguistique (Boyer, 2003 : 42). En outre, « toute représentation implique une évaluation, donc un contenu normatif qui oriente la représentation soit dans le sens d'une valorisation soit dans le sens d'une stigmatisation » (Boyer, 2001 : 42). Elles convoquent la notion de prestige elle-même liée à la langue et au dialecte qui peut être latent ou apparent. Le succès social d'une langue basée sur un dialecte s'explique par un prestige fort et apparent de ce dialecte (covert prestige vs overt prestige). La distinction entre langue et dialecte est davantage sociale et politique que linguistique.

Henri Boyer indique que les représentations se révèlent au travers de trois types de manifestations : "l'activité épilinguistique», définie comme une "activité discursive essentiellement de nature normative » concernant la langue et qui peut être " ordinaire, scolaire ou médiatique », "les pratiques métalinguistiques » qui correspondent à la production de dictionnaires, grammaires, ..., et « les interventions glottopolitiques » (Boyer, $2003: 44)$. La notion de conception d'une langue correspond à une approche plus conceptuelle que la notion de représentations.

Les prescriptions institutionnelles se voient donc traversées par une conception implicite de la langue qui vient empêcher, dans une certaine mesure, de répondre à l'injonction qu'elle promeut elle-même au métalangage, et qui devient dès lors paradoxale : la langue de l'école est une langue du commentaire, de la glose, au sein de laquelle usage et mention s'enchevêtrent; privilégier les formulations non ambigües en toutes circonstances, suggérant par là que cet enchevêtrement est le produit de mésusages de la langue, promouvoir le métalangage en écartant l'autonymie ne favorise pas le développement des compétences nécessaires aux apprentissages conceptuels. (Delarue-Breton, $2016: 28)$

\section{Méthodologie}

Pour proposer une analyse descriptive des représentations et conceptions et de la langue basque dans les instructions officielles, nous avons constitué un corpus d'analyse émanant du Ministère de l'Éducation nationale. Ce corpus distingue les textes réglementant l'enseignement des langues régionales en France (1976-2017), les programmes d'enseignement de langues 
régionales avec leurs déclinaisons pour le basque (2007-2019), les ressources d'accompagnement pour l'enseignement du basque en lycée (2011-2013) et les documents cadres pour l'organisation des enseignements dans les établissements bilingues du $1^{\text {er }}$ degré (2011 et 2018). Ils mettent en lumière les attendus de la demande ministérielle quant à l'enseignement de la langue basque dans les écoles, collèges et lycées en Pays basque de France.

Le corpus d'analyse se compose de textes relatifs à l'enseignement des langues régionales depuis 1976. La circulaire 76-123 du 29 mars 1976 parue au BOEN n ${ }^{\circ} 14$ le 8 avril 1976 propose une prise en compte dans l'enseignement des patrimoines culturels et linguistiques français. La circulaire 2001-166 dite " Circulaire Lang » du 5 septembre 2001 parue aux BOEN n $33 \mathrm{du}$ 13 septembre 2001 traite du développement de l'enseignement des langues régionales à l'école, au collège et au lycée. La circulaire 2001-167 du 5 septembre 2001 parue au BOEN n³3 le 13 septembre 2001 a été modifiée par l'Arrêté du 12 avril 2003 paru au BOEN n²4 du 12 juin 2003 et fixe les modalités de mise en place de l'enseignement bilingue à parité horaire par arrêté du 30 mai 2003. La brochure ministérielle Apprendre et enseigner les langues et les cultures régionales dans l'école de la République publiée par le Ministère de l'Éducation nationale en 2013 présente un état des lieux de l'enseignement des langues régionales en France. L'arrêté interministériel du 15/03/2017 fixe les sections et les modalités d'organisation des concours de l'agrégation « Section langues de France »; il est paru dans le Journal officiel de la République française $n^{\circ} 0070$ du 23 mars 2017. Enfin, la circulaire n²017-072 du 12-4-2017 relative à l'enseignement des langues et cultures régionales fixe la place des langues régionales dans la politique nationale de l'apprentissage des langues vivantes, les modalités de l'enseignement optionnel et bilingue et les orientations d'une politique d'enseignement des langues et cultures régionales.

À ces textes règlementaires viennent s'ajouter les programmes nationaux de langue vivante régionale $(2003,2007,2010,2019)$, les programmes de langue basque pour le primaire (2007), les programmes de langue basque option pour le collège (2007 et 2010), les ressources d'accompagnement pour l'enseignement de la langue basque au lycée (2011 et 2013), les documents cadres pour l'organisation des enseignements dans les établissements bilingues du $1^{\text {er }}$ degré (2011 et 2018). Les textes sont présentés dans l'ordre chronologique :

L'arrêté du 30 mai 2003 publié dans le Bulletin officiel hors-série n², 19 juin 2003 fixe un programme pour les langues étrangères et régionales à l'école primaire. Le Bulletin officiel hors-série $\mathrm{n}^{\circ} 9$ du 27 septembre 2007 présente des programmes de l'enseignement de langues régionales pour l'école primaire, avec une introduction commune et une annexe pour le basque. Le Bulletin officiel spécial $\mathrm{n}^{\circ} 4$ du 29 avril 2010 publie un programme d'enseignement des langues vivantes pour la classe de seconde générale et technologique. Le programme d'enseignement pour le basque pour le pallier 2 du collège parait dans le Bulletin officiel $n^{\circ} 27$ du 8 juillet 2010. En 2011, le document cadre pour l'organisation des enseignements dans les établissements bilingues du $1^{\text {er }}$ degré est proposé par une équipe de conseillers pédagogiques dirigée par l'inspectrice de l'Éducation nationale en charge de la langue basque. En octobre 2011, le Ministère de l'Éducation nationale publie des ressources pour la classe de seconde générale et technologique, des thèmes d'étude et des fiches thématiques succinctes. En avril 2013, des ressources pour le cycle terminal proposent des exemples de sujets d'étude.

Le Bulletin officiel n² du 26 mars 2015 entré en vigueur à la rentrée 2015 est consacré aux programmes de maternelle ${ }^{2}$. Le Bulletin officiel $n^{\circ} 39$ du 20 octobre 2015 intitulé « Enseignement des Langues Vivantes Étrangères et Régionales » et le Bulletin officiel $n^{\circ} 11$ du 26 novembre 2015 intitulé «Programmes d'enseignement de l'école élémentaire et du collège » sont pris en considération. Le Document cadre pour l'organisation des enseignements

\footnotetext{
${ }^{2}$ Les enseignements sont organisés en cinq domaines d'apprentissage : Mobiliser le langage dans toutes ses dimensions ; Agir, s'exprimer, comprendre à travers l'activité physique ; Agir, s'exprimer, comprendre à travers les activités artistiques ; Construire les premiers outils pour structurer sa pensée ; Explorer le monde.
} 
dans les établissements bilingues du $1^{\text {er }}$ degré (2011 et 2018) fait partie du corpus d'étude. Enfin, les programmes de langues, littératures et cultures régionales - basque - de première générale et de Terminale générale (2019) sont de récents documents également intégrés dans le corpus choisi.

\section{Résultats}

\section{Représentations de la langue basque dans les instructions officielles}

Le programme de 2010 pour le palier 2 du collège ( $4^{\text {ème }}$ et $\left.3^{\text {ème }}\right)$ soumet une liste indicative d'œuvres littéraires ou un ensemble de contenus artistiques, mais une grande liberté pédagogique est accordée aux enseignants.

La liste des contenus culturels ci-dessous est indicative et non exhaustive. Elle ne cherche à établir ni une hiérarchie ni une progression. Il reviendra au professeur d'opérer dans ces entrées possibles des choix cohérents et diversifiés $d u$ début à la fin du palier 2. (2010)

Le programme de collège et de lycée est centré sur les notions culturelles : il s'agit d'en parcourir l'histoire officielle et les genres canoniques, plus que d'enrichir une relation au monde et aux mots par un réel travail de langage.

\section{D’une représentation patrimoniale à une visée utilitariste}

En 1976, la circulaire de prise en compte dans l'enseignement des patrimoines culturels et linguistiques français met en évidence une représentation patrimoniale et figée des langues régionales de France.

Ne reconnaitre l'existence d'un tel patrimoine culturel qu'en certaines zones où se trouve parlée la langue locale serait nier une réalité partout tangible et appauvrir une richesse nationale.

Chaque fois qu'une langue est pratiquée sous forme de dialectes différenciés, c'est le dialecte correspondant au lieu où l'enseignement est dispensé et la graphie la plus appropriée à ce dialecte qui seront utilisés? (1976)

En 1982, la circulaire dite Savary est une avancée majeure pour l'enseignement des langues régionales au sein de l'Éducation nationale car elle organise les enseignements. L'autorisation des expérimentations d'ouvertures de classes bilingues permet la création et l'institutionnalisation de sections français-langues régionales à l'école publique et l'enseignement des langues et cultures régionales peut être considéré comme une matière spécifique. Les conditions dans lesquelles pourraient être créées des classes expérimentales bilingues sont étudiées en tenant compte des expériences déjà engagées dans certaines régions et en faisant appel aux compétences révélées.

En 1995, la circulaire dite Bayrou renforce la mise en place d'un enseignement bilingue qui « commence dès le cycle 1 et se poursuit à l'école élémentaire. La langue régionale y est à la fois langue enseignée et langue d'enseignement». "Le bilinguisme se définit par un enseignement à parité horaire entre la langue régionale et la langue française ». Un enseignement d'une ou deux disciplines est proposé dans la langue régionale au collège.

En 2001, la circulaire dite Lang prolonge officiellement le cursus bilingue français - langue régionale jusqu'au lycée général et professionnel. 
L'article L. 312-10 du code de l'éducation a réaffirmé la possibilité de dispenser un enseignement des langues et cultures régionales tout au long de la scolarité dans les régions où celles-ci sont en usage. L'éducation nationale se doit de faire vivre ce patrimoine culturel, de veiller au développement des langues régionales et de contribuer à leur transmission. Oublier cette responsabilité ne serait pas un signe de modernité. Ce serait au contraire une perte de substance de l'héritage culturel national. (Pascaud et Viaut, 2017)

En 2007, 1'approche communicative fait ainsi son apparition dans le programme de basque du palier 1. L'objectif de l'apprentissage de la langue basque et les pratiques associées se caractérisent par cet aspect communicatif de la langue basque. Les activités de communication proposent le développement d'une langue quotidienne, pouvant parfois être au détriment des activités de pensée et de réflexion. Après l'adoption du CECRL en 2004, c'est l'ensemble des programmes de langues vivantes qui a été réécrit (Bonet, 2007).

En 2011, les ressources de seconde générale et technologique associent les niveaux du CECRL aux genres textuels scolaires académiques issus des pratiques sociales de référence normées. La description à l'écrit et à l'oral, le débat, le résumé d'un article de presse, le panneau d'affichage, la rédaction d'un texte argumentatif sont autant d'exemples de tâches. Ces ressources proposent de «(B1) Faire un exposé à l'oral sur une compétition maritime, finale de trainières de la baie de Saint-Sébastien avec description des équipages et compte-rendu de la course » ou d' " (B1) Organiser un débat sur la gestion des ressources halieutiques : pour ou contre la pêche industrielle mondialisée, la pisciculture marine...» ou avoir comme objectif d' « être capable de prendre part à un débat sur des thèmes liés à la ville » (2011).

En 2017, l'arrêté interministériel fixant les sections et les modalités d'organisation des concours de l'agrégation ajoute la « Section langues de France » à la « Section langues vivantes étrangères » pour les options «basque, breton, catalan, corse, créole, occitan-langue d'oc, tahitien ». Dans ce texte portant sur la création de l'agrégation pour une liste de «langues de France $»$, le champ sémantique de ces dernières interfère ainsi avec celui des langues régionales du ministère de l'Éducation nationale (Pascaud et Viaut, 2017). Les langues de France sont considérées comme constituant un patrimoine linguistique visant à enrichir l'identité culturelle de la France.

Une grammaire de phrase

Entre 2007 et 2010, les programmes de basque de primaire et de collège conçus sur le modèle de ceux de langues vivantes étrangères proposent une grammaire de phrase associée aux compétences du CECRL en réception, production et interaction $(2007,2010)$.

Réception

L’impératif, le potentiel; les subordonnées relatives; les déictiques; l'interrogation directe et indirecte; les complétives en -teko / -tzeko; le cas instrumental; les repères temporels :-ondoren [après], -aitzin [avant]- eta gero... ; les cas ablatif, adlatif, inessif. - nondik - nora ; la relative substantivée; quelques formes de Nor-nori; quelques formes de Nor-nori-nork; les interjections; le tutoiement; quelques formes allocutives; quelques formes de Nor-nork; les formes du passé ; l'aspect : bukatua / ez bukatua [accompli/non accompli] ; formes simples de nor-nori au passé ; la relative; les adverbes de temps ; les marqueurs de temps : l'expression de la simultanéité, de l'antériorité et de la postériorité ; les circonstancielles de temps; l'ordre et la défense ; la phrase négative ; l'expression du but; le conditionnel; les subordonnées interrogatives indirectes ; le partitif; le nom verbal ; quelques formes simples du potentiel; le 
passé simple; valeur de l'imparfait et du passé simple (ekintza bukatua eta bukatugabea [action accomplie et non-accomplie]); les causales; les concessives; sobera (gehiegi) / -egi [trop]; les comparatives; la phrase elliptique ;

Ainsi, cette liste dresse des compétences morphosyntaxiques à acquérir par les élèves en langue basque.

Les ressources d'accompagnement de seconde générale et du cycle terminal (2011 et 2013) privilégient l'approche communicative et notionnelle et proposent un seul objectif grammatical en morphologie verbale. «Être capable d'employer à bon escient les temps du passé, du présent et du futur en cohérence avec les thèmes d'étude » (2011). En 2015, il n'existe pas de déclinaison linguistique ni culturelle pour les langues régionales alors que des repères de progressivité sont proposés pour les langues étrangères (espagnol, allemand, anglais, italien, arabe, chinois, portugais).

En 2019, en l'absence de repères de progressivité pour le basque, le centre pédagogique associatif IKAS propose plusieurs fiches grammaticales pour les enseignants de basque mais transposables aux élèves ${ }^{3}$ et issues d'une grammaire de phrase dont nous avons relevé quelques exemples ci-dessous :

L'expression du temps et de l'espace, postposition arte vs morphème -raino; l'expression de la similarité bera vs berdina ; le complément du nom-ren vs -ko; la calque syntaxique -(r)entzat; les locutions verbales Behar / Nahi / Gogo izan [devoir/vouloir/avoir envie] ; le préfixe ba- ; Badu / Duela ; Bere / Haren ; Berri / beste ; les verbes synthétiques; Dago, Dauka; le morphème interrogatif indirect Duenez / duenetz ; les conjonctions Edo / [ou bien] ; Ere, Baita... ere [aussi], Ezta... ere [si ce n'est] ; expression de l'impersonnel; les propositions subordonnées comparatives; l'indéfini; expression de la mesure; le cas partitif; les propositions subordonnées relatives; la distinction des pronoms interrogatifs zer et zein [que et quel].

On retrouve des similitudes entre les points grammaticaux abordés.

\section{Langue des disciplines, approche métalinguistique et comparative}

La répartition disciplinaire en basque et en français proposée pour l'organisation des enseignements dans les établissements bilingues du $1^{\text {er }}$ degré est une proposition de mise en œuvre des enseignements (2011 et 2018). Comme l'indique l'article 2 de l'arrêté du 12 mai 2003 « aucune discipline ou aucun domaine disciplinaire, autre que la langue régionale, ne peut être dispensé exclusivement en langue régionale ». Ainsi, les domaines disciplinaires tels que l'éducation physique et sportive, la pratique artistique et l'histoire des arts et la découverte du monde sont dispensés pour la moitié du temps d'enseignement en langue basque. Aux cycles 2 et 3 , les mathématiques sont un domaine disciplinaire où la langue d'enseignement est majoritairement la langue basque alors que le domaine disciplinaire de la langue est dispensé majoritairement en langue française. L'orthographe fait son apparition en 2018 alors qui n'était pas dispensé en langue basque en 2011. En 2018, l'enseignement moral et civique au cycle 2 est proposé en langue basque.

Les programmes du collège et le nouveau socle commun de connaissances, de compétences et de culture mettent l'accent sur les langages et leurs modes de fonctionnement. Le programme de langues vivantes de 2015 souligne l'importance du fonctionnement d'un système linguistique d'une langue vivante régionale :

\footnotetext{
${ }^{3}$ Centre pédagogique IKAS
} 
Ce domaine considère les langages moins dans leur usage que dans le principe de leur acquisition. Il appelle la mise en place de procédures de mémorisation, d'entrainement, d'automatisation et de réflexion sur les objets qu'il travaille, et au premier chef sur la langue française.(...) L'enseignement des langues étrangères ou régionales permet d'étendre et de diversifier ses capacités de compréhension et d'expression écrites et orales dans plusieurs langues; de passer d'un mode de communication à un autre; de recourir à divers moyens langagiers pour interagir et apprendre; de réfléchir sur les fonctionnements des langues, leurs variations internes, leurs proximités et distances.

Le développement de la compréhension du fonctionnement d'un système linguistique au collège peut être vu comme les premiers pas vers une « approche raisonnée » de la langue telle qu'elle est préconisée dans les programmes du lycée (cf. BO spécial n ${ }^{\circ} 4$ du 29 avril 2010). En effet, le programme de 2015 repris dans le document cadre affirme l'importance de la compréhension du fonctionnement d'un système linguistique de la langue vivante régionale :

Ce domaine considère les langages moins dans leur usage que dans le principe de leur acquisition. Il appelle la mise en place de procédures de mémorisation, d'entrainement, d'automatisation et de réflexion sur les objets qu'il travaille, et au premier chef sur la langue française. (...)

L'enseignement des langues étrangères ou régionales permet d'étendre et de diversifier ses capacités de compréhension et d'expression écrites et orales dans plusieurs langues ; de passer d'un mode de communication à un autre ; de recourir à divers moyens langagiers pour interagir et apprendre; de réfléchir sur les fonctionnements des langues, leurs variations internes, leurs proximités et distances.

L'observation comparée de quelques phénomènes simples dans des langues différentes (dont la langue française) crée chez les élèves une distance qui leur permet d'être plus sensibles aux réalités grammaticales et renforce la maitrise du langage.

En section bilingue, l'approche contrastive est systématisée et permet de façon très positive d'introduire dans le cursus, dès le cycle 2, une langue vivante étrangère. (Académie de Bordeaux, 2011)

\section{Littérature de jeunesse et littérature classique}

Les représentations de la langue basque dans les programmes sont étroitement liées à la littérature et au choix des œuvres complètes qui y sont proposées. Les œuvres intégrales du programme de collège de 2010 ont pour la plupart été primées lors de prix de littérature de jeunesse au Pays basque et en Espagne. Elles sont bien souvent traduites en plusieurs langues - anglais, espagnol, français, catalan, russe, japonais, etc. - ou proposent pour certaines d'entre elles des éditions trilingues. Le programme recense des recueils de poésies de Kirmen Uribe Bitartean heldu eskutik [En attendant, donne-moi la main] $(2001)^{4}$, prix de la critique de la poésie écrite en langue basque, de Juan Kruz Igerabide, Begi-niniaren poemak, [Les poèmes des prunelles] (1999) recueil composé de haikus japonais et Hosto Gorri, Hosto Berde [Feuille rouge, feuille verte]. Les œuvres en prose s'intitulent Kokodriloa Ohe Azpian [Le crocodile sous le lit] (2002) de Mariasun Landa qui a été lauréate du prix national d'Espagne de littérature de jeunesse en 2003 ou Battita Handia (1993) de Daniel Landart ouvrage en langue basque unifiée et marqué par des tournures propres au dialecte navarro-labourdin littéraire et lauréat du prix de la ville d'Irun, Irun saria. La série Banbuloren Istorio Banbulotarrak (2000-2006)

\footnotetext{
${ }^{4}$ Les références bibliographiques des œuvres littéraires se trouvent dans le programme (MEN, 2010).
} 
de Bernardo Atxaga met en scène un chien qui montre la vérité aux humains, le rôle joué par les chiens et comment les chiens ont pu être relégués par la société. D'autres œuvres littéraires comme Nire eskua zurean [Ma main dans la tienne] (1995) de Mariasun Landa, Urtziren Diarioa [Le journal d'Urtzi] (2015) d'Antton Kazabon, Lamia [La lamie] (1998) de Josu Lartategi, Risky, zaude geldirik ! [Risky, reste tranquille!] (2001) de Jon Iturralde font également leur entrée dans les programmes, de même que l'intégrale de la bande dessinée d'aventure maritime Justin Hiriart (Muro Harriet). Ce tour d'horizon des ouvrages littéraires met en évidence des œuvres originales de littérature de jeunesse contemporaine ayant pour la plupart d'entre elles été primées et une absence d'œuvres de littérature classique adaptées.

Les œuvres et des textes littéraires inclus dans le programme d'enseignement de spécialité de Première (MEN, 2019) dénotent une variété plus importante au lycée pour un public de spécialistes, comparativement au programme de collège. Quatre des six dialectes littéraires recensés par le dialectologue Koldo Zuazo (2014 : 71) sont présents, sans compter la langue littéraire unifiée contemporaine. Parmi les œuvres de littérature classique, on trouve le chef d'œuvre Guero (1643) de Pedro Axular en dialecte littéraire labourdin côtier, le poème mythique Azken oyua (1937) d'Esteban Urkiaga Lauaxeta en dialecte biscayen littéraire, le recueil de fables Alegiak (1926) de Jules Moulier Oxobi en dialecte navarro-labourdin littéraire, les poèmes «Goizean goizik jeiki nündüzün » [Je me levai à l'aube] de Lohitegi (1640), " Atharratze Jaurgainean » [Au château de Tardets] (1870) et «Bortüetako elürra » [La neige des montagnes] (1950) de Bordazarre Etxahun en dialecte souletin littéraire; Leturiaren egunkari ezkutua (1957) premier roman moderne basque de la littérature basque de J.L. Alvarez «Txillardegi » met en scène les questions existentielles du personnage Leturia; Zergatik panpox (1979) d'Arantxa Urretabizkaia a été traduit en espagnol, en catalan et en bulgare.

Parmi les œuvres contemporaines, on rencontre le chef d'œuvre Obabakoak (1988) de Bernardo Atxaga traduit dans plus de vingt langues qui a obtenu le prix national espagnol de la narration en 1989, le roman Gorde nazazu lurpean [Garde-moi sous terre] (2000) de Ramon Saizarbitoria, le roman Twist (2011) d'Arkaitz Cano qui a obtenu le Prix Euskadi 2012 dans la catégorie « Littérature en langue basque », Musshe (2012) de Kirmen Uribe traduit en japonais et classé pour l'année 2015 en $5^{\text {ème }}$ position aux côtés d'auteurs comme Michel Houllebecq, David Mitchell ou Kazuo Ishiguro. Rappelons que Kirmen Uribe a reçu le prix national de littérature espagnole et le Prix de la Critique 2008 pour le roman basque Bilbao-New YorkBilbao.

\section{Variété linguistique}

Le programme de langues vivantes en seconde de 2010 intègre la variation linguistique à travers la notion de registre et de types de textes appliquées à l'oral et à l'écrit. Elle est appréhendée tant dans une approche diachronique et historique de la langue que synchronique.

\section{Registres et évolution}

Les élèves sont entrainés à reconnaitre et à utiliser, à bon escient, les divers registres de la langue: soutenu/familier; littéraire/journalistique... et à différencier les codes de l'écrit et de l'oral, qui connaissent des écarts souvent considérables. Le recours éventuel à des textes plus anciens permet aussi de prendre conscience de l'évolution historique de la langue. (2010)

Les schémas très simples mentionnés ci-dessus comme par exemple la distinction écrit/oral, impliquent des représentations schématiques de la langue associant registre soutenu et langue écrite et registre familier et langue orale. De même, la diversité dialectale est mise en perspective ouvrant des possibilités à l'utilisation des dialectes : 


\section{Standard et variétés}

Même si les normes de la langue conventionnellement considérée comme standard restent la référence, les élèves sont aussi confrontés à des documents variés de par leur origine géographique et sociale, qui peuvent comporter des particularismes marqués, voire des réalisations dialectales, qui font la richesse des langues. La variation porte sur le lexique, sur des traits phonologiques, morphologiques, ou syntaxiques, qui peuvent différer fortement. Cela peut représenter une difficulté particulière pour la compréhension à laquelle les élèves sont petit à petit familiarisés, en reconnaissance. Ils sont invités à relever les traits spécifiques et à identifier la variété linguistique que ces traits caractérisent, éventuellement à en faire usage ponctuellement en situation. Cet entrainement leur ouvre plus largement les portes des sociétés, par les échanges directs dans la communication spontanée, le contact avec les ouvres vivantes (chanson, roman, cinéma ...) qui font sa place à l'expression populaire. (2010)

Les ressources d'accompagnement du basque font apparaitre une absence de cette variété linguistique dans les thèmes d'étude qui par ailleurs est présente dans les fiches thématiques à travers la variation dialectale (2011). La notion de registres n'y est pas exploitée. L'objectif " Avoir une approche des variantes dialectales actuelles sur les régions côtières (ce qui change et ce qui ne change pas » (2011) vise une distinction des caractéristiques des dialectes littéraires côtiers, biscayen, guipuscoan et labourdin côtier. En effet, le dialecte littéraire labourdin côtier du XVII ${ }^{\text {ème }}$ siècle a laissé place au dialecte littéraire navarro-labourdin au $\mathrm{XX}^{\text {ème }}$ siècle, dont la grammaire a été fixée par Pierre Lafitte (Lafitte, 1944). De même, il peut s'agir de caractéristiques phonologiques ou syntaxiques de ces dialectes (solécisme côtier, conjugaison de l'auxiliaire avoir à deux actants au lieu de trois arraina eman nau vs arraina eman dit). L'objectif «Identifier les différents dialectes sur les divers éléments syntaxiques et lexicaux » (2011) fait référence aux particularismes des cinq dialectes en présence aujourd'hui (Zuazo, 2014).

Le programme spécifique à l'enseignement de spécialité basque de première de 2019 reprend la représentation historique de la langue basque présente dans les programmes de 2010. L'axe intitulé « Une littérature en quête de norme linguistique » met en relation une tradition littéraire relativement récente et l'histoire de la langue. La langue basque est appréhendée dans une approche diachronique et historique, afin de donner aux élèves une culture linguistique qui intègre l'histoire de la langue basque.

L'un des principaux problèmes auxquels ont été confrontés les auteurs depuis le début de la littérature basque, autant en France qu'en Espagne, est celui de l'orthographe. Les langues voisines dont la production littéraire est plus ancienne (latin, occitan, espagnol et français) ont servi de modèles aux écrivains et il fallut attendre le début $d u X X^{e}$ siècle pour qu'une réflexion collective commence à définir des règles linguistiques communes, dans un domaine linguistique basque fortement marqué par la présence de dialectes et soumis à des systèmes graphiques différents. Cette réflexion aboutit à la création de l'Académie basque (Euskaltzaindia en 1919) et à la naissance d'une langue littéraire unifiée (Euskara batua en 1968). Le premier axe de cette thématique amène les élèves à rechercher et comprendre les choix orthographiques des différents auteurs à travers l'étude de textes écrits à la même époque (par exemple en basque et en latin au XVI siècle). Une étude comparative peut également être menée autour des choix lexicaux et des changements phonologiques au cours des siècles, selon les zones d'influence. C'est l'occasion de les analyser en fonction des contextes géopolitiques (en 
s'interrogeant sur le mythe de la "pureté de la langue " qui s'impose à la fin du $X I X^{e}$ siècle), et du pragmatisme de la production de masse dans la société moderne. (2019)

\section{Vers une conception de la discipline scolaire « basque »}

Il est important de distinguer la discipline « basque » constituée de la « langue basque » des « lettres basques » et de la « culture basque » et le basque des disciplines, où la langue est une compétence transversale pour l'enseignement de l'ensemble des disciplines. Dans les filières bilingues, la langue est donc à la fois vecteur et objet d'enseignement, le moyen d'accéder à des savoirs disciplinaires. La discipline « basque » doit être pensée et enseignée à la fois comme une langue vivante et comme une discipline littéraire, culturelle et artistique. Des questions se posent : dans quelle mesure la langue de scolarisation doit faire référence au standard et à la variété linguistique ? L'orthographe, totem de la langue française est-elle substituée par la grammaire en ce qui concerne le basque ? Ainsi, pour illustrer cette conception plurielle de la langue basque scolaire, nous proposons des orientations pour les programmes de grammaire de cycle 3 et 4, ainsi que des ressources pour l'enseignement de la culture littéraire et artistique basque au cycle 3 .

\section{Éléments pour une grammaire scolaire plurinormaliste du basque}

Comparé au lexique, à la phonologie ou à l'orthographe, la composante grammaticale a bien souvent une fonction normative très marquée. La connaissance de la grammaire basque est fondamentale pour l'apprentissage de la langue. Sans remettre en cause l'importance de la norme notamment dans le cas des langues minorées, une grammaire scolaire plurinormaliste est-elle envisageable ? Cette conception de langues et grammaires plurinormées a été inventée par le sociolinguiste Jean Baptiste Marcellesi dans les années 1980. Il considère que toute langue est nécessairement plurinormée ; à partir de l'exemple corse, les langues polynomiques sont considérées comme des "langues dont l'unité est abstraite et résulte d'un mouvement dialectique et non de la simple ossification d'une norme unique, et dont l'existence est fondée sur la décision massive de ceux qui la parlent de lui donner un nom particulier et de la déclarer autonome des autres langues reconnues » (Marcellesi, 1983 :314). Les utilisateurs d'une langue polynomique lui «reconnaissent plusieurs modalités d'existence, toutes également tolérées sans qu'il n'y ait entre elles hiérarchisation ou spécialisation de fonction. Elle s'accompagne de l'intertolérance entre utilisateurs de variétés différentes sur les plans phonologiques et morphologiques... » (Marcellesi, 1983 : 170). Cette même notion a été reprise plus tard dans les années 1990 (Vargas, 1996).

Le programme pourrait s'appuyer sur une grammaire scolaire située entre la grammaire descriptive très exhaustive et qui définit l'ensemble des phrases grammaticales et la grammaire normative plutôt partielle, pour proposer une association de plusieurs types de grammaires : une grammaire traditionnelle historique, une grammaire notionnelle-fonctionnelle développée par l'approche communicative et une grammaire textuelle ${ }^{5}$. Pour s'éloigner d'une conception univoque et figée de la langue basque diffusée par l'école, une grammaire plurinormée pourrait être envisageable.

Les principales caractéristiques typologiques de la langue basque auraient toute leur place dans la partie grammaticale du programme de basque : ordre des mots Sujet-Objet-Verbe, ergativité, langue agglutinante et post positionnelle, présence et accord des actants dans l'auxiliaire, transitivité et intransitivité des verbes, alternance des auxiliaires entre structures passives et actives, langue pro-drop, la notion de la seule marque casuelle portée par le dernier

\footnotetext{
${ }^{5} \mathrm{La}$ grammaire textuelle basque pourrait intégrer les résultats des recherches récentes effectuées au Pays basque Sud sur les genres textuels en langue basque.
} 
élément du groupe nominal, etc. Rappelons que les deux principales langues scolaires des élèves sont typologiquement éloignées.

En morphologie verbale, les propositions suivantes ajoutent une conception plurielle à la grammaire proposée par les programmes : valeur de l'aspect verbal (accompli pour le récit, non accompli pour la description), approche de la valeur des temps à travers l'emploi des verbes (le récit au passé à la troisième personne, le discours au présent ou au passé composé), absence de la marque de l'objet de référence dans l'auxiliaire (kaierra eman dut irakasleari [j'ai donné le cahier au professeur]), radical verbal au potentiel, subjonctif, impératif (pentsa daiteke, ikus dezan, jar zaitez [on peut penser, pour qu'il voie, asseyez-vous], distinction des deux auxiliaires de l'indicatif et du subjectif, potentiel, impératif, conjugaison allocutive vouvoyée ${ }^{6}$ (idazle hau lapurtarra duxu [cet écrivain est labourdin], la variante de l'auxiliaire avoir à trois actants (arkatza hartzen dakot [je lui prends une crayon]), etc. En morphologie nominale, les variantes orientales auraient également toute leur place. En outre, l'observation des marques du nombre (l'indéfini en plus du singulier et du pluriel), l'absence de genre grammatical, l'identification des classes de mots qui se déclinent ou dérivent (nom, verbe, déterminant, adjectif, pronom) seraient à prendre en considération.

Les registres auraient également une place de choix dans les programmes et une grammaire scolaire du basque. Le registre soutenu se manifeste notamment à l'écrit à partir de modèles littéraires, le registre familier se situe au niveau d'un code commun. De même, les registres (réaliste, fantastique, épique, lyrique, tragique, comique, etc.) seraient à intégrer, même si le public d'élèves des sections bilingues français-basque a le basque comme langue seconde.

Ainsi, la production de matériel pédagogique qui par définition pousse à la norme fait apparaitre deux sous-normes pour la langue basque scolaire au Pays basque de France : la sous norme souletine et la sous norme navarro-labourdine. Cette normalisation linguistique par l'école s'inscrit dans un contexte plus large de revitalisation linguistique de la langue basque dans la société.

\section{Éléments pour une culture littéraire et artistique}

En linguistique historique, les élèves découvrent la variation interne de la langue écrite au plan diachronique grâce à l'étude des états de langue du passé (Casenave et Idiazabal, à paraitre). En dialectologie, ils ont accès aux variations dialectales à travers les œuvres classiques écrites dans les dialectes littéraires, biscayen, guipuscoan, souletin, labourdin et basnavarrais (Zuazo, 2014 : 71). L'intérêt linguistique des corpus littéraires se caractérise par une appréhension de la production littéraire de l'ensemble de l'aire bascophone. D'un point de vue culturel, les corpus permettent un accès à une culture différente, comme par exemple les proverbes qui font apparaitre le monde de la nature et de la vie à la campagne aux élèves urbains. Le patrimoine littéraire constitue donc un fonds intéressant pour transmettre une langue enrichie de son épaisseur historique et d'un large ensemble de ses registres disponibles (Casenave et Idiazabal, à paraitre).

Dans le domaine disciplinaire de la culture littéraire et artistique basque au cycle 3 , il n'existe pas de ressource institutionnelle qui propose d'indications bibliographiques pour chaque entrée du programme. Voici quelques pistes de lecture d'albums, de bandes dessinées, de roman et récits illustrés, de contes et fables qui correspond à une conception élargie et variée de la langue basque scolaire.

Pour l'entrée «Héros et personnages », les extraits de romans Piarres (1926) de Jean Barbier, le récit de mœurs Pilota partida [La partie de pelote] (1910) de Jean Etchepare, les bandes dessinées d'Asisko comme Azti hitza [Le mot du devin] (2018) consacrée au personnage

\footnotetext{
${ }^{6}$ Les pratiques d'enseignants proposent des solutions empiriques, avec intégration des formes allocutives dans certaines écoles.
} 
Agosti Chaho et Maria Zugarramurdikoa eta sorginduak izan ziren emakumeak [Marie de Zugarramurdi et les femmes ensorcelées] (2018) de Mindegia et Petrirena consacrée aux sorcières sont autant de pistes de lecture. Pour l'entrée "Vivre des aventures ", il est possible d'envisager des extraits du recueil de contes d'Aingeru Epaltza Lur zabaletan [Vastes terres] (1994), de l'adaptation de la fiction de Pio Baroja Zalakain abenturazalea [L'aventurier Zalakain] (2006) ou de la bande dessinée d'Olazagasti et Yaguna, Everest euskal espedizioa [L'expédition basque Everest] (2018). Pour l'entrée « La morale en questions », de nombreuses fables de La Fontaine sont traduites, adaptées ou réécrites en basque par des auteurs comme Bizenta Moguel, Jean Baptiste Archu, Martin Goyhetche, Zaldubi, Jules Moulier Oxobi, etc. (Casenave, 2018). De même, le roman Haur besoetakoa 1954 de Jon Mirande a été adapté en bande dessinée (2018) par Unai Iturriaga. Pour l'entrée "Se confronter au merveilleux », l'album Otsoa, axeria eta ahuntzak [Le loup, le renard et les chèvres] (Hennebutte, 2009), les contes des recueils Ipar Euskal Herriko ipuinak eta leiendak [Les contes et légendes du Pays basque Nord] (2015) rassemblés par Jean-François Cerquand et republiés par Anuntxi Arana, Baigorriko zazpi liliak [Les sept fleurs de Baigorry] (1886) de Jean Pierre Duvoisin et Ixtoriomisterio [Histoires et mystères] (1929) de Jean Barbier sont autant de pistes de lecture. Pour l'entrée "Se découvrir, s'affirmer dans le rapport aux autres », les récits autobiographiques dans Aihen ahula [Le sarment faible] (1978) de Daniel Landart ou les contes Muntra, [La montre] Gauez gau [D'une nuit à l'autre] (1967) de Jean Etchepare Landerretche proposent une approche linguistique navarro-labourdine. Pour l'entrée "Célébrer le monde » les poèmes Ikusten duzu goizean [Vous voyez le matin] (Elizanburu, 1857), ou ceux du recueil poétique Hinki hanka (1979) de Manex Erdozaintzi Etxart ${ }^{7}$ sont des extraits et œuvres incontournables.

\section{Conclusion}

L'analyse des instructions officielles a mis en évidence de nombreuses représentations autour la langue basque comme discipline scolaire, langue que nous pourrions qualifier à la fois de patrimoniale, communicative, métalinguistique, utilitaire, etc. Nous avons vu qu'elle peut être perçue non seulement comme une langue de communication mais également comme une langue de prestige, de savoir, de culture et de littérature à la fois classique, historique, moderne et contemporaine. Cette analyse pourrait permettre d'alimenter une réflexion autour de la création d'outils spécifiques adaptés à un public d'élèves pour qui la langue basque est la langue seconde, en leur permettant d'acquérir la langue et la culture basque dans de bonnes conditions.

D'autres recherches sont nécessaires pour définir les usages, les représentations et les conceptions de la langue basque : une analyse de productions d'élèves permettrait par exemple de mettre en avant les variables linguistiques de la situation d'écriture et une didactique de l'écriture (Doquet, 2012). Une étude axée sur les pratiques des enseignants permettrait une mise en perspectives des programmes, une recherche menée en formation des enseignants, sur les conceptions présentes dans les récents mémoires de Master MEEF basque, etc. Par ailleurs, il serait également intéressant d'étudier les représentations et conceptions de la langue française dans le discours institutionnel à propos des langues régionales.

\footnotetext{
${ }^{7}$ Les textes et œuvres proposées par le centre pédagogique IKAS peuvent constituer des pistes de lecture pour les ressources d'accompagnement du cycle 3 .
} 


\section{Bibliographie}

AGRESTI Giovanni \& TURI Joseph-G, 2016, Représentations sociales des langues et politiques linguistiques. Déterminismes, implications, regards croisés. Actes du Premier Congrès mondial des droits linguistiques, vol. ${ }^{\mathrm{er}}$, Rome : Aracne.

BONET Luc, 2007, « Les sections bilingues français - langue régionale et le cadre européen : nouvelles perspectives didactiques », Tréma, n`28, p. 115-124.

BOYER Henri, 2001, Introduction à la sociolinguistique, Paris : Dunod, coll. " Les Topos ».

BOYER Henri, 2003, De l'autre côté du discours : recherches sur le fonctionnement des représentations communautaires, Editions L'Harmattan.

CASENAVE Jean et IDIAZABAL Itziar, (à paraitre), «L'usage des petits genres littéraires dans la formation des enseignants de langue basque », Presses universitaires de Rennes.

CASENAVE Jean, 2018, «Éléments pour l'élaboration d'un manuel de littérature basque pour les collèges et lycées », Lengas [Online], $\mathrm{n}^{\circ} 83$.

COYOS Jean-Baptiste, 2012, "L'enseignement de la langue basque en France. Essai d'évaluation de son impact dans la société », in Louis-Jacques Dorais et Abdallah El Mountassir (dirs.), L'enseignement des langues vernaculaires : défis linguistiques, méthodologiques et socio-économiques, L'Harmattan, Paris, p.17-44.

DELARUE-BRETON Catherine, 2016, Inégalité d'accès au savoir, ou inégalité d'accès au questionnement?, Note de synthèse d'HDR, Université Paris 8 Vincennes Saint Denis.

DOQUET Claire, 2012, « La norme et l'usage. Linguistique et didactique de l'écriture à l'école primaire », Repères, ${ }^{\circ} 46$, p. 95-109.

EUSKALTZAINDIA, 1985-2011, Euskal Gramatika Lehen Urratsak I-II-III-IV-V-VI-VII. Iruñea-Pamplona : Bilbo.

LABOV William, 1976 [édition originale 1972], Sociolinguistique, Paris : Les Éditions de Minuit, coll. « Le sens commun ».

LAFITTE Pierre, 1979 [1944], Grammaire Basque: Navarro-Labourdin Littéraire. $2^{\text {ème }}$ édition, San Sebastián / Bayonne : Elkar / Ikas.

LUDI Georges et PY Bernard, 2003 [1986], Être bilingue, Bern: Peter Lang, coll. « Exploration. Recherches en sciences de l'éducation ».

MARCELLESI Jean Baptiste, 1983, «"Langues régionales” et pédagogie ou comment assumer le concept de langue polynomique ? », in Petitjean A. et Romian H. (dirs.), Recherches actuelles sur l'enseignement $d u$ français, Actes du $\mathrm{II}^{\mathrm{e}}$ Colloque international de didactique et pédagogie du français. Paris : INRP, p. 200-202.

PASCAUD Antoine et VIAUT Alain, 2017, «Pour une définition de la notion de "langue régionale" », Lengas [En ligne], n82, consulté le 12 avril 2020.

VARGAS Claude, 1996, "Grammaire et didactique plurinormaliste du français », Repères, Recherches en didactique du français langue maternelle, $n^{\circ} 14$, p. 83-103.

ZUAZO Koldo, 2014, Euskalkiak, Elkar. 


\section{GLOTTOPOL}

Revue de sociolinguistique en ligne

Comité de rédaction : Michaël Abecassis (University of Oxford), Salih Akin (Université de Rouen Normandie), Sophie Babault (Université de Lille), Aude Bretegnier (Université du Mans), Claude Caitucoli, Véronique Castellotti (Université de Tours), Régine DelamotteLegrand (Université de Rouen Normandie), Alexandre Duchêne (Université de Fribourg), Valentin Feussi (Université d'Angers), Robert Fournier (Carleton University, Ottawa), Stéphanie Galligani (Université Grenoble Alpes), Médéric Gasquet-Cyrus (Université AixMarseille), Emmanuelle Huver (Université de Tours), Normand Labrie (Université de Toronto), Foued Laroussi (Université de Rouen Normandie), Benoit Leblanc (Université du Québec à Trois-Rivières), Mylène Lebon-Eyquem (Université de la Réunion), Fabienne Leconte (Université de Rouen Normandie), Gudrun Ledegen (Université de Rennes), Danièle Moore (Simon Fraser University, Vancouver), Clara Mortamet (Université Jean Monnet, Saint Etienne), Alioune Ndao (Université Cheik Anta Diop, Dakar), Isabelle Pierozak (Université de Tours), Cécile Van den Avenne (Université Sorbonne Nouvelle, Paris 3).

Rédactrice en chef : Clara Mortamet.

Directrice de publication : Fabienne Leconte.

Comité scientifique: Michelle Auzanneau (Université de Paris), Margaret Bento (Université de Paris), Jacqueline Billiez (Université de Grenobles Alpes), Philippe Blanchet (Université de Rennes), Jean-Michel Eloy (Université d'Amiens), Françoise Gadet (Université Paris Nanterre), Monica Heller (Université de Moncton), Caroline Juillard (Université de Paris), Jean-Marie Klinkenberg (Université de Liège), Marinette Matthey (Université Grenoble Alpes), Marie-Louise Moreau (Université de Mons-Hainault), Robert Nicolaï (Université Côte d'Azur), Didier de Robillard (Université de Tours), Valérie Spaëth (Université Sorbonne nouvelle), Claude Truchot (Université de Strasbourg), Daniel Véronique (Université AixMarseille).

\section{Comité de lecture pour ce numéro :}

Nathalie Auger, Michèle Auzanneau, Margaret Bento, Stéphane Bonnery, Josiane Boutet, Lucile Cadet, Danièle Cogis, Claudine Garcia-Debanc, Marc Debono Régine Delamotte, JeanFrançois De Pietro, Marie-Laure Elalouf, Valentin Feussi, Laurent Gajo, Emmanuelle Huver, Christian Lagarde, Mylène Lebon-Eyquem, Fabienne Leconte, Gudrun Ledegen, Nadia Maillard, Maira Mamede, Bruno Maurer, Clara Mortamet, Fanny Rink, Valerie Spaëth, Marielle Rispail, Françoise Ropé, Eguzki Urteaga, Cécile Van den Avenne, Daniel Véronique 